\title{
Seed Priming Improves the Germination Traits of Tall Fescue (Festuca arundinacea)
}

\author{
Hossein Reza ROUHI ${ }^{1}$, Mohammad Ali ABOUTALEBIAN² ${ }^{2}$, Farzad SHARIF-ZADEH ${ }^{1}$ \\ ${ }^{1}$ University of Tehran, Faculty of Agricultural Engineering and Technology, College of Agriculture and Natural Resources, Department \\ of Agronomy and Plant Breeding, Karaj, Islamic Republic of Iran; hosseinroobi@alumni.ut.ac.ir (corresponding author) \\ ${ }^{2}$ University of Bu-Ali Sina, Faculty of Agriculture, Department of Agronomy and Plant Breeding, Hamedan, Islamic Republic of Iran
}

\begin{abstract}
A couple of experiments were carried out to investigate the effects of osmopriming and hydropriming treatments on the germination indexes and seed vigor of Tall fescue (Festuca arundinacea). In the first experiment, fulfilled based on randomized complete block designed with four replicates, the effect of polyethyleneglycol 6000 solution with five osmotic potentials $(-8,-10,-12,-14$ and -16 bar), two temperatures $\left(15\right.$ and $\left.25^{\circ} \mathrm{C}\right)$ and four time intervals $(12,24,36$ and 48 hours) was analized. The second experiment was fulfilled by using distilled water at two temperatures $\left(15\right.$ and $\left.25^{\circ} \mathrm{C}\right)$ and four time intervals $(12,24,36$ and 48 hours) with four replicates on the above mentioned data. Then, based on the maximum germination rate and vigor indexes, the optimal treatment composition was determined for osmopriming and hydropriming solutions in regard to the osmotic potential, temperature and priming duration. In osmopriming experiment, treatment composition of 12 hours at $15^{\circ} \mathrm{C}$ and -8 bar had the most significant effect on the percent and speed of germination, length of radicle, coleoptile and seedling, as well as vigor indexes in Tall fescue. For hydropriming, the effect analyzed in the same condition as osmopriming, was observed in the majority of evaluated traits; treatment composition of 12 hours prime and $15^{\circ} \mathrm{C}$ had the maximum numerical value in comparison to control and other treatments. Finally, it is inferred that favorable effects of osmoand hydropriming at $15^{\circ} \mathrm{C}$ temperature are more significant in comparison with the control group (none prime seeds).
\end{abstract}

Keywords: germination, hydropriming, osmopriming, temperature, seed

\section{Introduction}

Rapid seed germination and stand establishment are critical factors for crop production under stress conditions. In many crop species, seed germination and early seedling growth are the most sensitive stages to stresses. Seed priming is known as the seed treatment which improves seed performance under environmental conditions (Ashraf and Foolad, 2005). In fact, seed priming is a procedure that partially hydrates the seed, after which the seeds are dried, this determing the germination process to start, but with no radicle emergence. Methods of seed priming have been described comprehensively by Bradford (1986) and Khan (1992) who include soaking seed in water or osmotic solution, and intermixture with porous matrix material. Lots of information is available, showing the hydration of seeds up to, but not exceeding, the lag phase with priming increased RNA and protein synthesis (Fu et al., 1988), faster embryo growth (Dahal et al., 1990) and reduced leakage of metabolites (Styer and Cantliffe, 1983) compared with control group. Seed priming has been found a doable technology to enhance rapid and uniform emergence, high vigor, and better yields for vegetable and flower species (Dearman et al., 1987; Parera and Cantliffe, 1994; Bruggink et al., 1999) small seeded grasses (Heydecker and Coolbaer, 1978; Bradford, 1986) and some field crops (Hartz and Caprile, 1995; Chiu et al.,
2002; Giri and Schillinger, 2003; Basra et al., 2005, 2006; Kaur et al., 2005; Kaya et al., 2006; Janmohammadi et al., 2009; Rouhi et al., 2011).

Seed priming is commonly used to reduce the time between seed sowing and seedling emergence (Parera and Cantliffe, 1994). Earlier works show that the success of seed priming is influenced by the complex interaction of factors including plant species, water potentiality of the priming agent, duration of priming, temperature, seed vigor and dehydration, as well as storage conditions of the primed seed (Parera and Cantliffe, 1994). Although, the previous studies indicate that some benefits are associated with pre-sowing treatments for seed vigor enhancement, there is a dearth of information about the germination traits of primed seeds of Tall fescue. Therefore, the present study was carried out considering the objective of evaluating the effects of different priming treatments on seed germination, manner of Tall fescue, under different temperature, in order to find out the most effective priming composition treatments.

\section{Materials and methods}

\section{Sample preparation and treatment}

This study was carried out at the Department of Agronomy, Faculty of Agriculture, University of Tehran, Iran. Tall fescue seeds were used as seed material and were 
58

obtained from the Natural Resources Organization of Gorgan (from Golestan province, Iran).

For osmopriming, Tall fescue seeds were immersed in osmotic potentials of $-8,-10,-12,-14,-16$ bar, for polyethylene glycol (PEG 6000) at $15^{\circ} \mathrm{C}$ and $25^{\circ} \mathrm{C}$ for $12,24,36$ and $48 \mathrm{~h}$ under dark conditions (Michel and Kaufmann, 1973). Thereafter, the seeds were rinsed with distilled water three times. The treated seeds were surface-dried and dried back to their original moisture content via experience at room temperature. Osmoprimed treated seeds was equilibrated at room temperature (about $25^{\circ} \mathrm{C}$ ) for 24 hours.

For hydropriming, seeds were immersed in distilled water at $15^{\circ} \mathrm{C}$ and $25^{\circ} \mathrm{C}$ for $12,24,36$ and $48 \mathrm{~h}$ under dark conditions. The treated seeds were surface-dried and dried back to their original moisture content at room temperature (about $25^{\circ} \mathrm{C}$ ) for 24 hours.

\section{Germination tests}

Four replicates of 50 seeds were put to germinate on top of double layered papers with $5 \mathrm{ml}$ of water in $9 \mathrm{~cm}$ Petri dishes. These Petri dishes with the seeds were put into sealed plastic bags to avoid moisture loss. Seeds were allowed to germinate at $15-25 \pm 1^{\circ} \mathrm{C}$ for 14 days (ISTA, 1996). Germination was considered to have occurred when the radicles were $2 \mathrm{~mm}$ long. Germination percentage was recorded every $12 \mathrm{~h}$ until final mentioned days. Germination rate was calculated as described in following formula (ISTA, 1996):

$\mathrm{GR}=\frac{\text { No. of germinated seed at first count }}{\text { Days of first count }} \ldots . . . \frac{\text { No. of germinated seeds at first count }}{\text { Days of final count }}$

Seedling length and seedling dry weight were measured after the $14^{\text {th }}$ days. Vigor indexes land 2 were calculated according to the following formula:

Vigor index $1(\mathrm{VI} 1)=$ [seedling length $(\mathrm{cm}) \times$ germination percentage]

Vigor index $2($ VI2 $)=$ [seedling dry weight $(\mathrm{g}) \times$ germination percentage]

\section{Statistical analysis}

The Statistical analysis was based on a randomized complete blocks design (RCBD); with four replications and 50 seeds per replicate. Data for germination percentage were subjected to arcsine transformation before the analysis of variance was carried out with SAS software.
Mean comparison was performed with Duncan's test if $\mathrm{F}$ test was significant at $(P<0.05)$.

\section{Results and discussion}

\section{Optimization of Tall fescue seed priming}

In order to determine the effects of priming treatments on germination characteristics, the first analysis of variance was performed for germination data without a control group. Significant three-way interactions (time, temperature and osmotic potential) were found for all investigated characters (data not shown). Secondly, the analysis of variance was performed for germination data with the control group.

In the first experiment, which was done in order to evaluate the best osmotic potential level, as well as osmopriming duration and temperatures, the variance analysis of data showed that all of the germination traits of Tall fescue seeds are affected by osmopriming treatments (Tab. 1 and Tab. 3).

Variance analysis of the second experiment was investigated in order to study hydropriming effect on Tall fescue seed under different intervals (12, 24, 36 and $48 \mathrm{~h}$ ) and two temperatures $\left(15\right.$ and $\left.25^{\circ} \mathrm{C}\right)$; it showed that germination percentage and some of the measured traits are effected by hydropriming in comparison with the control group (Tab. 2 and Tab. 4).

\section{Final Germination Percentage (FGP)}

In the osmopriming treatment, the maximum amount of final germination percentage was for the interval of 36 $\mathrm{h}$, potential of $-10 \mathrm{bar}$ and temperature of $15^{\circ} \mathrm{C}$, which did not have significant differences compared with the time of 12 and $24 \mathrm{~h}$, at the same temperature. But it was different within the interval of $48 \mathrm{~h}$ and other potentials in this time and at the same temperature (Tab. 3). The recommended time treatment is $12 \mathrm{~h}$. Superior treatment $(12 \mathrm{~h} \times-8 \mathrm{bar}$ $\times 15^{\circ} \mathrm{C}$ ) was more than control group. Demir and Van de Vanter (1999) reported that osmopriming of watermelon seeds caused the decrease of the mean germination time and the increase of its percentage.

For the hydropriming treatment, the maximum amount of this trait was for the interval of $12 \mathrm{~h}$, and temperature of $15^{\circ} \mathrm{C}$ (Tab. 4). Moradi et al. (2008) showed that, for most evaluated germination parameters of corn seeds, hydropriming was the effective treatment.

Tab. 1. The ANOVA table showing the osmopriming treatment in comparison with control on germination traits of Tall fescue

\begin{tabular}{ccccccccccc}
\hline \multicolumn{10}{c}{ Mean of squares $(\mathrm{MS})$} \\
\hline S.O.V & df & FGP $(\%)$ & GR $(1 /$ day $)$ & CL $(\mathrm{cm})$ & RL $(\mathrm{cm})$ & SL $(\mathrm{cm})$ & SDW $(\mathrm{gr})$ & VI1 & VI2 \\
\hline Block & 3 & $203.18^{\mathrm{ns}}$ & $0.0000076^{\mathrm{ns}}$ & $2.36^{\mathrm{ns}}$ & $0.048^{\mathrm{ns}}$ & $3.01^{\mathrm{ns}}$ & $0.0000013^{\mathrm{ns}}$ & $568^{\mathrm{ns}}$ & $0.11^{\mathrm{ns}}$ \\
Treatment & 40 & $1095.85^{* *}$ & $0.000046^{* *}$ & $6.2^{* *}$ & $5.9^{* *}$ & $21.97^{* *}$ & $0.0000044^{* *}$ & $3137^{* *}$ & $0.55^{* *}$ \\
Error & 120 & 42.37 & 0.0015 & 0.35 & 2.21 & 2.2 & 0.0000013 & 157 & 0.03 \\
\hline
\end{tabular}

ns, ${ }^{* *},{ }^{*}$ Respectively non significant and significant of 1 and 5 percent of probability; FGP : Final Germination Percentage; GR: Germination Rate; CL: Coleoptile Length; RL: Radicle Length; SL: Seedling Length; SDW: Seedling Dry Weight; VI1: Vigor Index 1; VI2: Vigor Index 2 
Tab. 2. The ANOVA table showing the hydropriming treatment in comparison with control on germination traits of Tall fescue

\begin{tabular}{cccccccccc}
\hline \multicolumn{7}{c}{ Mean of squares $(\mathrm{MS})$} \\
\hline S.O.V & df & FGP $(\%)$ & GR $(1 /$ day $)$ & CL $(\mathrm{cm})$ & RL $(\mathrm{cm})$ & SL $(\mathrm{cm})$ & SDW $(\mathrm{gr})$ & VI1 & VI2 \\
\hline Block & 3 & $3.86^{\mathrm{ns}}$ & $0.0000072^{\mathrm{ns}}$ & $0.36^{\mathrm{ns}}$ & $0.11^{\mathrm{ns}}$ & $0.07^{\mathrm{ns}}$ & $0.00000031^{\mathrm{ns}}$ & $26^{\mathrm{ns}}$ & $0.03^{\mathrm{ns}}$ \\
Treatment & 8 & $4218.55^{* *}$ & $0.00051^{* *}$ & $32.29^{* *}$ & $11.64^{* *}$ & $78.62^{* *}$ & $0.0000074^{* *}$ & $9997^{* *}$ & $2.37^{* *}$ \\
Error & 24 & 4.47 & 0.00000094 & 0.13 & 0.13 & 0.44 & 0.0000003 & 36 & 0.004 \\
\hline
\end{tabular}

$\mathrm{ns}^{* *},{ }^{*}$ Respectively non significant and significant of 1 and 5 percent of probability; FGP: Final Germination Percentage; GR: Germination Rate; CL: Coleoptile Length; RL: Radicle Length; SL: Seedling Length; SDW: Seedling Dry Weight; VI1: Vigor Index 1; VI2: Vigor Index 2

\section{Germination Rate (GR)}

In the osmopriming treatment, the maximum amount of germination rate was in the potential of -8 bar for 12 $\mathrm{h}$ at $15^{\circ} \mathrm{C}$ and it did not have significant differences with the treatment combination of $36 \mathrm{~h}$, potential of -10 bar at the same temperature. So, potential of -8 bar in the time of $12 \mathrm{~h}$ is recommended because of the lower time needed and high potential. Moreover, superior treatment was more than control group (Tab. 3). Hur (1991) showed that in Italian ryegrass and sorghum, germination percentage, as well as germination rate, increased in response to osmopriming.

In the hydropriming treatment, treatments for all time intervals at in $15^{\circ} \mathrm{C}$ were higher than at $25^{\circ} \mathrm{C}$, and the maximum amount of germination rate was in the case of $12 \mathrm{~h}$, and temperature of $15^{\circ} \mathrm{C}$ (Tab. 4). Harris et al. (1999), Giri and Schilinger (2003) and Finch-Savage et al. (2004) reported that the priming effect on germination rate was positive in comparison with control group.

\section{Coleoptile Length (CL)}

In the osmopriming treatment, the longest coleoptile was observed in the interval of $36 \mathrm{~h}$, potential of $-8 \mathrm{bar}$ and temperature of $15^{\circ} \mathrm{C}$. This temperature had higher amount of time and potential in comparison with the one at $25^{\circ} \mathrm{C}$. So, it can be said that the most suitable treatment combination is at $15^{\circ} \mathrm{C}$, potential of -8 bar and $12 \mathrm{~h}$, which was more than control group (Tab. 3). In a study with Turkish pine (Pinus brutia) var. eldarica, Khalil et al. (1997) determined that plants raised from seed preconditioned at room temperature in aerated solution of PEG 8000 for different time periods exhibited faster germination and higher shoot length compared to plants raised from untreated seed.

In the hydropriming treatment, the longest coleoptile was observed in the interval of $12 \mathrm{~h}$ and temperature of $15^{\circ} \mathrm{C}$, which was higher than control group (Tab. 4). Tomato seed primed resulted in significant increases in stem length, shoot weight, leaf area, number of flowers, fruit set, and final yield (Khalil and Moursy, 1983).

\section{Radicle Length (RL)}

In the osmopriming treatment, on this trait, because of the existence of several superior treatment combinations, the first treatment which is higher than control group and includes $15^{\circ} \mathrm{C}, 12 \mathrm{~h}$, potential of $-8 \mathrm{bar}$ is recommended
(Tab. 3). Osmoconditioning promoted the rates of radicle extension, seedling emergence,, expansion of the cotyledons and the first leaf of cucumber (Passam and Kakouriotis, 1994).

In the hydropriming treatment, the maximum radicle length was in the interval of $24 \mathrm{~h}$ with $15^{\circ} \mathrm{C}$ and it did not have significant differences compared with treatment combination of $12 \mathrm{~h}$ at the same temperature. So, it seems that the time interval of $12 \mathrm{~h}$ is recommended because of a lower time (Tab. 4). Priming has been shown to induce nuclear DNA synthesis in the radicle tip cells in tomato (Liu et al., 1997).

\section{Seedling Length (SL)}

In the osmopriming experiment, the analysis of treatments, including the control group, the temperature of $15^{\circ} \mathrm{C}$ proved optimal, showing higher results, which regarding to the economic aspect can be introduced as the superior treatment, beside the potential of -8 bar and time interval of $12 \mathrm{~h}$ (Tab. 2). Osmoconditioning of Bermuda grass (Cynodon dactylon) seed using PEG followed by immediate sowing improved germination and seedling growth under saline conditions (Al-Humaid, 2002).

In the hydropriming treatment, all seedling had lengths higher at the temperature of $15^{\circ} \mathrm{C}$ than at $25^{\circ} \mathrm{C}$, but the time interval of $12 \mathrm{~h}$ and $24 \mathrm{~h}$ at $15^{\circ} \mathrm{C}$ did not have significant differences. So, the interval of $12 \mathrm{~h}$ is recommended because of the lower time and preserve seeds from electrolyte leakage (Tab. 4). In snap beans (Phaseolus vulgaris), hydropriming resulted in improved germination and seedling emergence and growth (Suzuki and Khan, 2001).

\section{Seedling Dry Weight (SDW)}

In the osmopriming treatment, on this trait, regarding the optimal use of time and material, it was the time interval of $12 \mathrm{~h}$, at temperature of $15^{\circ} \mathrm{C}$ and potential of $-12 \mathrm{bar}$ that showed higher amounts in comparison with control group and other treatments (Tab. 3). Khalil et al. (1997) showed that plants raised from osmoprimed seed exhibited higher dry weight in comparison with control group. Similar positive effect of osmopriming on dry weight in Italian ryegrass (Lolium multiflorum) and sorghum (Sorghum bicolor) was observed (Hur, 1991). Dabrowska et al. (2001) determined that both solid matrix priming and osmopriming significantly increased the speed's capability of emergence and mean dry weight of hot pepper seedlings. 
Tab. 3. Effect of osmopriming treatments on the germination and seedling characteristics of Festuca arundinacea

\begin{tabular}{|c|c|c|c|c|c|c|c|c|c|c|}
\hline \multicolumn{3}{|c|}{ Treatments } & \multicolumn{8}{|c|}{ Traits } \\
\hline $\begin{array}{c}\text { Temperature } \\
\text { (Celsius) }\end{array}$ & $\begin{array}{l}\text { Time } \\
\text { (hours) }\end{array}$ & $\begin{array}{c}\text { Potential } \\
\text { (bar) }\end{array}$ & $\begin{array}{l}\text { FGP } \\
(\%)\end{array}$ & $\begin{array}{c}\text { GR } \\
\text { (1/day) }\end{array}$ & $\begin{array}{l}\mathrm{CL} \\
(\mathrm{cm}) \\
\end{array}$ & $\begin{array}{l}\mathrm{RL} \\
(\mathrm{cm})\end{array}$ & $\begin{array}{l}\mathrm{SL} \\
(\mathrm{cm})\end{array}$ & $\begin{array}{c}\text { SDW } \\
\text { (gr) }\end{array}$ & VI1 & VI2 \\
\hline \multirow{20}{*}{15} & \multirow{5}{*}{12} & -8 & $68.90 \mathrm{bc}$ & $0.016 \mathrm{a}$ & 7.71a-f & $5.13 \mathrm{a}-\mathrm{f}$ & $12.84 a b c$ & $0.025 \mathrm{a}$ & $884.6 \mathrm{~cd}$ & $1.72 \mathrm{bc}$ \\
\hline & & -10 & $65.38 \mathrm{bcd}$ & $0.015 b c$ & $7.12 \mathrm{~d}-\mathrm{h}$ & $5.84 \mathrm{abc}$ & $12.96 a b c$ & $0.021 \mathrm{e}-\mathrm{k}$ & $847.3 \mathrm{de}$ & $1.37 \mathrm{~b}-\mathrm{e}$ \\
\hline & & -12 & $68.62 b c$ & $0.015 b c$ & $7.14 \mathrm{~d}-\mathrm{h}$ & $4.72 \mathrm{a}-\mathrm{h}$ & 11.86b-f & $0.024 a b$ & $813.8 \mathrm{ef}$ & $1.64 \mathrm{bcd}$ \\
\hline & & -14 & $65.54 \mathrm{bcd}$ & $0.0145 c$ & $8.36 \mathrm{ab}$ & $4.23 \mathrm{c}-\mathrm{k}$ & $12.59 \mathrm{a}-\mathrm{d}$ & $0.021 \mathrm{e}-\mathrm{k}$ & $825.1 \mathrm{e}$ & $1.37 \mathrm{~b}-\mathrm{e}$ \\
\hline & & -16 & $69.04 b c$ & $0.015 b c$ & $7.31 \mathrm{c}-\mathrm{g}$ & $5.1 \mathrm{a}-\mathrm{f}$ & $12.42 \mathrm{a}-\mathrm{e}$ & $0.023 \mathrm{a}-\mathrm{d}$ & $857.4 \mathrm{~d}$ & $1.58 \mathrm{bcd}$ \\
\hline & \multirow{5}{*}{24} & -8 & $62.13 \mathrm{~b}-\mathrm{e}$ & $0.014 \mathrm{bcd}$ & $8.22 \mathrm{abc}$ & $5.48 \mathrm{a}-\mathrm{e}$ & 13.70ab & $0.022 \mathrm{a}-\mathrm{g}$ & 851de & $1.36 \mathrm{~b}-\mathrm{f}$ \\
\hline & & -10 & 63.56b-e & $0.014 \mathrm{bcd}$ & 7.54b-f & $4.1 \mathrm{c}-\mathrm{k}$ & 11.63b-f & $0.021 \mathrm{e}-\mathrm{k}$ & 739.2gh & $1.33 \mathrm{~b}-\mathrm{e}$ \\
\hline & & -12 & $67.14 b c$ & $0.015 b c$ & $8.37 \mathrm{ab}$ & 6.19ab & $14.57 \mathrm{a}$ & $0.021 \mathrm{e}-\mathrm{k}$ & $977.6 \mathrm{~b}$ & $1.40 \mathrm{~b}-\mathrm{e}$ \\
\hline & & -14 & $65.14 \mathrm{bcd}$ & $0.014 \mathrm{bcd}$ & $7.8 \mathrm{a}-\mathrm{e}$ & $5.85 \mathrm{abc}$ & $13.65 \mathrm{ab}$ & $0.021 \mathrm{e}-\mathrm{k}$ & 888.6cd & $1.36 \mathrm{~b}-\mathrm{e}$ \\
\hline & & -16 & $67.28 b c$ & $0.015 b c$ & $7.98 \mathrm{a}-\mathrm{d}$ & $5.13 \mathrm{a}-\mathrm{f}$ & 13.10ab & $0.021 \mathrm{e}-\mathrm{k}$ & $881.3 \mathrm{bcd}$ & $1.41 \mathrm{bcd}$ \\
\hline & \multirow{5}{*}{ 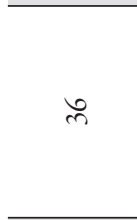 } & -8 & $62.07 \mathrm{~b}-\mathrm{e}$ & $0.013 \mathrm{~b}-\mathrm{e}$ & $8.58 \mathrm{a}$ & $4.68 \mathrm{a}-\mathrm{h}$ & $13.27 \mathrm{ab}$ & $0.02 \mathrm{f}-\mathrm{k}$ & $823.6 \mathrm{ef}$ & $1.24 \mathrm{~b}-\mathrm{f}$ \\
\hline & & -10 & $90 \mathrm{a}$ & $0.016 a b$ & $7.69 \mathrm{a}-\mathrm{f}$ & $5.57 \mathrm{a}-\mathrm{e}$ & 13.26ab & $0.022 \mathrm{a}-\mathrm{g}$ & $1193.4 \mathrm{a}$ & $1.98 \mathrm{a}$ \\
\hline & & -12 & $68.18 b c$ & $0.015 b c$ & 7.54b-f & $6.24 a$ & 13.78ab & $0.021 \mathrm{e}-\mathrm{k}$ & $938.4 b c$ & $1.43 \mathrm{~b}-\mathrm{e}$ \\
\hline & & -14 & $67.74 b c$ & $0.015 b c$ & $7.47 \mathrm{~b}-\mathrm{f}$ & $5.7 \mathrm{a}-\mathrm{d}$ & 13.17ab & $0.021 \mathrm{~d}-\mathrm{k}$ & $892.1 \mathrm{c}$ & $1.42 \mathrm{bcd}$ \\
\hline & & -16 & $71.08 \mathrm{~b}$ & $0.0155 \mathrm{~b}$ & $7.98 \mathrm{a}-\mathrm{d}$ & $5.7 \mathrm{a}-\mathrm{d}$ & $13.68 \mathrm{ab}$ & $0.0216 \mathrm{a}-\mathrm{i}$ & $972.3 \mathrm{~b}$ & $1.53 \mathrm{bcd}$ \\
\hline & \multirow{5}{*}{48} & -8 & 61.96b-e & $0.013 \mathrm{~b}-\mathrm{e}$ & $7.69 \mathrm{a}-\mathrm{f}$ & $5.27 \mathrm{a}-\mathrm{e}$ & $12.97 \mathrm{abc}$ & $0.0213 c-j$ & $803.6 \mathrm{f}$ & $1.31 \mathrm{~b}-\mathrm{f}$ \\
\hline & & -10 & $64.90 \mathrm{bcd}$ & $0.014 \mathrm{bcd}$ & $6.81 \mathrm{e}-\mathrm{i}$ & $4.88 \mathrm{a}-\mathrm{g}$ & $11.69 \mathrm{~b}-\mathrm{f}$ & $0.021 \mathrm{e}-\mathrm{k}$ & $758.7 \mathrm{~g}$ & $1.36 \mathrm{~b}-\mathrm{e}$ \\
\hline & & -12 & 63.18b-e & $0.013 \mathrm{~b}-\mathrm{e}$ & $6.81 \mathrm{a}-\mathrm{d}$ & $4.53 a-j$ & $11.34 \mathrm{~b}-\mathrm{f}$ & $0.023 \mathrm{a}-\mathrm{d}$ & $716.5 \mathrm{~h}$ & $1.45 \mathrm{f}-\mathrm{i}$ \\
\hline & & -14 & $65.82 \mathrm{bcd}$ & $0.014 \mathrm{bcd}$ & $7.98 \mathrm{a}-\mathrm{d}$ & $5.21 \mathrm{a}-\mathrm{f}$ & 13.19ab & $0.021 \mathrm{e}-\mathrm{k}$ & $868.1 \mathrm{~cd}$ & $1.38 \mathrm{~b}-\mathrm{e}$ \\
\hline & & -16 & $68.93 b c$ & $0.015 b c$ & $7.72 \mathrm{a}-\mathrm{f}$ & $5.11 \mathrm{a}-\mathrm{f}$ & $12.84 a b c$ & $0.0214 \mathrm{~b}-\mathrm{j}$ & $884.6 \mathrm{~cd}$ & $1.47 \mathrm{bcd}$ \\
\hline \multirow{21}{*}{25} & \multirow{5}{*}{12} & -8 & $66.18 \mathrm{bcd}$ & $0.014 \mathrm{bcd}$ & $6.76 \mathrm{f}-\mathrm{i}$ & $5.54 a-e$ & $12.3 \mathrm{a}-\mathrm{f}$ & $0.021 \mathrm{e}-\mathrm{k}$ & $814.0 \mathrm{ef}$ & $1.38 \mathrm{bcd}$ \\
\hline & & -10 & $50.39 \mathrm{fg}$ & $0.0115 \mathrm{fg}$ & $6 \mathrm{i}-1$ & $4.31 \mathrm{~b}-\mathrm{j}$ & $10.32 \mathrm{~d}-\mathrm{h}$ & $0.021 \mathrm{e}-\mathrm{k}$ & $520 \mathrm{~m}$ & $1.05 \mathrm{e}-\mathrm{h}$ \\
\hline & & -12 & $61.53 \mathrm{~b}-\mathrm{e}$ & $0.013 \mathrm{~b}-\mathrm{e}$ & $6.18 \mathrm{~h}-\mathrm{k}$ & $4.3 b-j$ & $10.49 \mathrm{c}-\mathrm{h}$ & $0.0195 \mathrm{~g}-\mathrm{k}$ & $645 j$ & $1.19 \mathrm{bc}$ \\
\hline & & -14 & $24.14 \mathrm{kl}$ & $0.01 \mathrm{kl}$ & $4.03 p$ & $2.67 \mathrm{j}-\mathrm{m}$ & $6.71 \mathrm{k}$ & $0.021 \mathrm{e}-\mathrm{k}$ & $162 u$ & $0.50 \mathrm{k}$ \\
\hline & & -16 & $49.25 \mathrm{fg}$ & $0.011 \mathrm{fg}$ & $5.32 \mathrm{k}-\mathrm{o}$ & $3.3 \mathrm{f}-1$ & $8.615 \mathrm{~g}-\mathrm{j}$ & $0.02 \mathrm{f}-\mathrm{k}$ & 424.20 & $0.98 \mathrm{~d}-\mathrm{g}$ \\
\hline & \multirow{5}{*}{24} & -8 & $59.39 c-f$ & $0.0125 c-f$ & $6.21 \mathrm{~h}-\mathrm{k}$ & $3.8 \mathrm{~d}-1$ & $10.05 \mathrm{e}-\mathrm{i}$ & $0.021 \mathrm{e}-\mathrm{k}$ & $596.8 \mathrm{k}$ & $1.24 \mathrm{~b}-\mathrm{e}$ \\
\hline & & -10 & $55.55 \mathrm{def}$ & $0.012 \mathrm{~d}$ & $5.93 \mathrm{i}-1$ & $3.96 \mathrm{c}-\mathrm{k}$ & $9.89 \mathrm{f}-\mathrm{i}$ & $0.021 \mathrm{e}-\mathrm{k}$ & 549.31 & $1.16 \mathrm{c}-\mathrm{f}$ \\
\hline & & -12 & $66.15 \mathrm{bcd}$ & $0.014 \mathrm{bcd}$ & $6.38 \mathrm{~g}-\mathrm{j}$ & $3.96 \mathrm{c}-\mathrm{k}$ & $10.34 \mathrm{~d}-\mathrm{h}$ & $0.019 \mathrm{~h}-\mathrm{k}$ & $683.9 \mathrm{i}$ & $1.25 \mathrm{bcd}$ \\
\hline & & -14 & $31.69 \mathrm{ijk}$ & $0.0114 \mathrm{ijk}$ & $4.86 \mathrm{~m}-\mathrm{p}$ & $1.46 \mathrm{~m}$ & $6.323 \mathrm{k}$ & $0.018 \mathrm{k}$ & $200.3 t$ & $0.57 \mathrm{jk}$ \\
\hline & & -16 & $31.31 \mathrm{jk}$ & $0.0112 \mathrm{jk}$ & $5.45 \mathrm{j}-\mathrm{n}$ & $4.43 a-j$ & $9.89 \mathrm{f}-\mathrm{i}$ & $0.02 \mathrm{f}-\mathrm{k}$ & $310 \mathrm{r}$ & $0.62 \mathrm{jk}$ \\
\hline & \multirow{5}{*}{36} & -8 & 38.33hij & 0.0115hij & 4.69 nop & 2.9h-m & $7.59 \mathrm{ijk}$ & $0.02 \mathrm{f}-\mathrm{k}$ & $291 \mathrm{~s}$ & 0.76hij \\
\hline & & -10 & $53.30 \mathrm{ef}$ & $0.0121 \mathrm{ef}$ & $6.36 \mathrm{~g}-\mathrm{j}$ & $3.73 \mathrm{f}-1$ & $10.09 \mathrm{f}-\mathrm{i}$ & $0.02 \mathrm{f}-\mathrm{k}$ & $537.71 \mathrm{~m}$ & 1.06b-f \\
\hline & & -12 & 41.76gh & $0.012 \mathrm{ghi}$ & $5.031-\mathrm{o}$ & $2.36 \mathrm{klm}$ & 7.39e-h & $0.0185 \mathrm{jk}$ & $307 \mathrm{rs}$ & $0.77 \mathrm{~g}-j$ \\
\hline & & -14 & 19.381 & 0.00951 & 4.42op & $2.74 \mathrm{i}-\mathrm{m}$ & $7.17 \mathrm{jk}$ & $0.019 \mathrm{~h}-\mathrm{k}$ & $138.9 \mathrm{v}$ & $0.36 \mathrm{k}$ \\
\hline & & -16 & $23.57 \mathrm{kl}$ & $0.01 \mathrm{kl}$ & $5.52 \mathrm{j}-\mathrm{n}$ & $3.14 \mathrm{~g}-\mathrm{m}$ & $8.65 \mathrm{~g}-\mathrm{j}$ & $0.0182 \mathrm{ijk}$ & $203.8 \mathrm{t}$ & $0.42 \mathrm{k}$ \\
\hline & \multirow{5}{*}{48} & -8 & $37.72 \mathrm{hij}$ & 0.0115hij & $4.88 \mathrm{~m}-\mathrm{p}$ & $4.59 \mathrm{a}-\mathrm{i}$ & $9.475 \mathrm{~g}-j$ & $0.02 \mathrm{f}-\mathrm{k}$ & $357.4 p$ & $0.75 \mathrm{hij}$ \\
\hline & & -10 & $42.98 \mathrm{gh}$ & $0.0152 \mathrm{gh}$ & $5.71 \mathrm{j}-\mathrm{n}$ & $2.64 \mathrm{j}-\mathrm{m}$ & $8.35 \mathrm{~g}-j$ & $0.02 \mathrm{f}-\mathrm{k}$ & 358.11p & $0.85 \mathrm{~b}-\mathrm{e}$ \\
\hline & & -12 & $52.98 \mathrm{ef}$ & $0.0121 \mathrm{ef}$ & $5.43 \mathrm{j}-\mathrm{n}$ & $3.14 \mathrm{~g}-\mathrm{m}$ & $8.58 \mathrm{~g}-\mathrm{j}$ & $0.0182 \mathrm{ijk}$ & $454.5 n$ & $0.96 b-f$ \\
\hline & & -14 & 191 & 0.00951 & $4.79 \mathrm{~m}-\mathrm{p}$ & $1.98 \mathrm{lmn}$ & $6.783 \mathrm{k}$ & $0.02 \mathrm{f}-\mathrm{k}$ & $128.8 \mathrm{w}$ & $0.38 \mathrm{k}$ \\
\hline & & -16 & 35.76hij & 0.015 hij & 6.1ijk & $3.78 \mathrm{~d}-1$ & $9.89 \mathrm{f}-\mathrm{i}$ & $0.019 \mathrm{~h}-\mathrm{k}$ & $350.4 p q$ & $0.67 \mathrm{ij}$ \\
\hline & Control & & $55.12 \mathrm{def}$ & $0.0115 \mathrm{hij}$ & $7.27 \mathrm{c}-\mathrm{g}$ & $3.34 \mathrm{f}-1$ & 10.6ghi & $0.02 \mathrm{f}-\mathrm{k}$ & $584.2 \mathrm{k}$ & $1.1 \mathrm{~b}$ \\
\hline
\end{tabular}

In each column means followed by the same letter are not significantly different at the $P<0.05$ level; FGP : Final Germination Percentage; GR: Germination Rate; CL: Coleoptile Length; RL: Radicle Length; SL: Seedling Length; SDW: Seedling Dry Weight; VI1: Vigor Index1; VI2: Vigor Index2

In the hydropriming treatment, all of maximum amounts of this trait were observed at temperature of $15^{\circ} \mathrm{C}$. The interval of $12 \mathrm{~h}$ was better than other treatments at same temperature because it took higher dry weight, compared with the other treatments and control group (Tab. 4). In field experiments, hydropriming of safflower (Carthamus tinctorius) seeds for $12 \mathrm{~h}$ resulted in higher capitula per plant, grains per capitulum, 1000-seed weight, grain yield, and oil content compared to untreated seeds (Bastia et al., 1999). Similar improvements were ob- 
Tab. 4. Effect of hydropriming treatments on the germination and seedling characteristics of Festuca arundinacea

\begin{tabular}{|c|c|c|c|c|c|c|c|c|c|}
\hline \multicolumn{2}{|c|}{ Treatments } & \multicolumn{8}{|c|}{ Traits } \\
\hline $\begin{array}{l}\text { Temperature } \\
\text { (Celsius) }\end{array}$ & $\begin{array}{l}\text { Time } \\
\text { (hours) }\end{array}$ & FGP (\%) & GR (1/day) & $\mathrm{CL}(\mathrm{cm})$ & $\mathrm{RL}(\mathrm{cm})$ & $\mathrm{SL}(\mathrm{cm})$ & SDW (gr) & VI1 & VI2 \\
\hline \multirow{4}{*}{15} & 12 & $77.31 \mathrm{a}$ & $0.016 \mathrm{a}$ & $8.98 \mathrm{ab}$ & $5.43 a$ & $14.41 \mathrm{a}$ & $0.025 a$ & $1114 a$ & $1.93 \mathrm{a}$ \\
\hline & 24 & $65.54 \mathrm{~b}$ & $0.015 \mathrm{ab}$ & $8.73 b c$ & $5.48 \mathrm{a}$ & $14.21 \mathrm{a}$ & $0.023 \mathrm{ab}$ & $931.3 \mathrm{~b}$ & $1.53 \mathrm{~b}$ \\
\hline & 36 & $63 \mathrm{c}$ & $0.013 b c$ & $8.37 \mathrm{c}$ & $3.92 \mathrm{c}$ & $12.3 \mathrm{~b}$ & $0.023 \mathrm{ab}$ & $757.9 \mathrm{c}$ & $1.45 \mathrm{c}$ \\
\hline & 48 & $55.72 \mathrm{e}$ & $0.011 \mathrm{e}$ & $8.4 \mathrm{c}$ & $4.17 \mathrm{~b}$ & $12.57 \mathrm{~b}$ & $0.022 \mathrm{~b}$ & $700.4 \mathrm{~d}$ & $1.22 \mathrm{~d}$ \\
\hline \multirow{4}{*}{25} & 12 & $40.6 \mathrm{f}$ & $0.008 \mathrm{f}$ & $5.87 \mathrm{e}$ & $3.7 \mathrm{c}$ & $9.57 \mathrm{c}$ & $0.021 \mathrm{c}$ & $388.5 f$ & $0.85 f$ \\
\hline & 24 & $27.6 \mathrm{~g}$ & $0.005 \mathrm{~g}$ & $3.38 \mathrm{f}$ & $1.6 \mathrm{e}$ & $4.98 \mathrm{e}$ & $0.0211 \mathrm{c}$ & $137.5 \mathrm{f}$ & $0.58 \mathrm{~g}$ \\
\hline & 36 & $6.89 \mathrm{~h}$ & $0.001 \mathrm{~h}$ & $1.64 \mathrm{~h}$ & $0.23 \mathrm{f}$ & $1.87 \mathrm{~g}$ & $0.018 \mathrm{e}$ & $13 \mathrm{~h}$ & $0.125 \mathrm{~h}$ \\
\hline & 48 & $6.89 \mathrm{~h}$ & $0.001 \mathrm{~h}$ & $2.4 \mathrm{~g}$ & $1.37 \mathrm{e}$ & $3.77 \mathrm{f}$ & $0.02 \mathrm{~d}$ & $26 \mathrm{~g}$ & $0.13 \mathrm{~h}$ \\
\hline Control & & $58 \mathrm{~d}$ & $0.012 \mathrm{~d}$ & $7.03 \mathrm{~d}$ & $2.05 \mathrm{~d}$ & $9.08 \mathrm{~d}$ & $0.02 \mathrm{~d}$ & $526.6 \mathrm{e}$ & $1.16 \mathrm{e}$ \\
\hline
\end{tabular}

In each column means followed by the same letter are not significantly different at the P < 0.05 level; FGP: Final Germination Percentage; GR: Germination Rate; CL: Coleoptile Length; RL: Radicle Length; SL: Seedling Length; SDW: Seedling Dry Weight; VI1: Vigor Index 1; VI2: Vigor Index 2

served on maize, rice, chickpea (Harris et al., 1999), and pearl millet (Kumar et al., 2002) grown under dry land conditions.

\section{Vigor Index 1}

In the osmopriming treatment, the treatment combination of $36 \mathrm{~h}$, potential of $-10 \mathrm{bar}$, at $15^{\circ} \mathrm{C}$ can be regarded as superior in comparison with control group, by showing higher amounts (Tab. 3). Osmopriming may contribute to rapid seed germination by affecting active oxygen metabolism. In wild rye (Leymus chinensis) seeds, for example, priming with $30 \%$ PEG for $24 \mathrm{~h}$ resulted in an increase of the activity of superoxide dismutase (SOD) and peroxidase (POD) and a rapid increase of the respiratory intensity, which were associated with an increase of germination vigor (Jie et al., 2002).

In the hydropriming treatment, the maximum amount of this trait was in the time interval of $12 \mathrm{~h}$ and temperature of $15^{\circ} \mathrm{C}$, which has significant differences compared with the other treatments and control group (Tab. 4). Hydropriming resulted in a significant improvement of germination and seedling vigor and a decrease in leakage of electrolytes from germinating seeds (Srinivasan et al., 1999).

\section{Vigor Index 2}

In the osmopriming treatment, on this trait, superior treatment showed higher amount $\left(15^{\circ} \mathrm{C} \times 12 \mathrm{~h} \times-8 \mathrm{bar}\right)$ compared with control group (Tab. 3). Fu et al. (1988) determined that soaking peanut (Arachis hypogaea) seed in 20-25\% PEG for 48 h greatly increased phosphate uptake and RNA synthesis in embryonic axes while improving seeds' vigor.

In the hydropriming treatment, same as vigor index 1 , the maximum amount of this trait was in the time interval of $12 \mathrm{~h}$ at temperature of $15^{\circ} \mathrm{C}$, which has significant differences compared with the other treatments and control group (Tab. 4). Thornton and Powell (1992) determined that for seeds of cauliflower (Brassica oleracea) and Brussels sprouts (Brassica oleracea), an $8 \mathrm{~h}$ hydropriming treatment at $25^{\circ} \mathrm{C}$ were the most effective for improving the rate and uniformity of germination, root growth, and seed vigor.

\section{Conclusions}

During the osmopriming experiment upon this plant, time treatments of $12 \mathrm{~h}$ and $36 \mathrm{~h}$ allocated above amounts to themselves, and were superior to control group in the majority of investigated traits. Priming's temperature had an outstanding effect, since on all of the investigated traits, the temperature of $15^{\circ} \mathrm{C}$ was the best. It seems that the temperature of $15^{\circ} \mathrm{C}$ is near the optimum temperature of germination for this plant. So, it has significant effect on germination factors and indexes. Between the time intervals which are candidates of a superior treatment, the $12 \mathrm{~h}$ one can be introduced, because it can save time as well as prevent possible damages, like infection of seeds (because of seeds being in the solution for the longer time).

Researches explain that priming is a practical technique to increase germination rate and consistence, as well as vigor increase and a better performance in vegetables, flower plant and crops (Bruggink, 1999; Chiu et al., 2002; Farooq et al., 2008).

In hydropriming, time interval of $12 \mathrm{~h}$ and temperature of $15^{\circ} \mathrm{C}$ were superior than other treatments and control group. Zheng et al. (2002) reported that, at lower temperature, priming had significant effect on the germination rate of rice seeds. Shivankar et al. (2003) explained that hydropriming can increase germination rate and consistency in seeds with low viability. Singh et al. (1999) also conveyed the similar results. Effects of hydropriming on water potential, the driving force for water uptake during imbibitions, and the activity of $\alpha$-amylase were examined on wheat and rice kernels (Andoh and Kobata, 2002). Amylases are key enzymes that play a vital role in hydrolyzing the seed's starch reserve, thereby supplying sugars to the developing embryo is a must (Ashraf and Foolad, 2005). Hydropriming of cereal rye and perennial ryegrass 
62

can significantly increase the rate percentage of germination (Snap et al., 2008). Giri and Schilinger (2003) showed that the effect of hydropriming with water is equal, and in some cases even more significant, than other priming environments.

Regarding the positive effects of seed priming on germination characteristic of Tall fescue, it could be used as pre-sowing treatment in field conditions. In order to maintain a high quality of the primed seeds for extended storage periods, seeds should be stored at a low temperature and low moisture content, or after seed priming, seeds should be planted.

Finally it is recommended that the results of this study to be investigated in the farm condition in order to confirm the fulfilled experiments of this project.

\section{References}

Al-Humaid AI (2002). Effects of osmotic priming on seed germination and seedling growth of bermudagrass (Cynodon dactylon L.) under saline conditions. Bulletin of Cairo University 53:265-274.

Andoh H, Kobata T (2002). Effect of seed hardening on the seedling emergence and alpha amylase activity in the grains of wheat and rice sown in dry soil. Japanian J Crop Sci 71:220-225.

Ashraf M, Foolad MR (2005) Pre-sowing seed treatment a shotgun approach to improve germination, plant growth, and crop yield under saline and non-saline conditions. Adv Agron 88:223-271.

Basra SMA, Farooq M, Tabassum R (2005). Physiologycal and biochemical aspects of seed vigour enhancement treatments in fine rice (Oryza sativa L.). Seed Sci Technol 33:623-628.

Basra AS, Farooq M, Afzal I, Hussain M (2006). Influence of osmopriming on the germination and early seedling growth of coarse and fine rice. Int J Agric and Biol 8:19-21.

Bastia DK, Rout AK, Mohanty SK, Prusty AM (1999). Effect of sowing date, sowing methods and seed soaking on yield and oil content of rainfed saffower grown in Kalahandi, Orissa. Indian J Agron 44:621-623.

Bradford KJ (1986). Manipulation of seed water relations wia osmotic priming to improve germination under stress conditions. Hort Sci 21:1105-1111.

Bruggink GT, Ooms JJ, Van der Toorn P (1999). Induction of longevity in primed seeds. Seed Sci Res 9:49-53.

Chiu KY, Chen CL, Sung JM (2002). Effect of priming temperature on storability of primed sh-2 sweet corn seed. Crop Sci 42:1996-2003.

Dabrowska B, Suchorska Tropilo K, Capecka E (2001). Presowing conditioning of hot pepper (Capsicum annuum L.) seeds and its results in a field growing. Part I. Effect on the vigour of seeds and seedlings, p. 3-7. In Annals Warsaw Agriculture University (Landscape Architecture).

Dahal P, Bradford KJ, Jones RA (1990). Effects of priming and endosperm integrity on seed germination rates of tomato genotypes. II. Germination at reduced water potential. J Experimental Bot 41:1441-1453.

Dearman J, Brocklehust PA, Drew RLK(1987). Effect of osmotic priming and aging on the germination and emergence of carrot and leek seed. Annual Ap Biol 111:717-722.

Demir J, Van de Venter HA (1999). The effect of priming treatments on the performance of watermelon Citrollus Lanatns (Thunb) Matsun Nakai seeds under temperature and osmotic stress. Seed Sci Technol 27:871-875.

Farooq M, Basra MA, Wahid A, Cheema ZA, Cheema MA, Khaliq A (2008) Physiological Role of Exogenously Applied Glycinebetaine to Improve Drought Tolerance in Fine Grain Aromatic Rice (Oryza sativa L.). J Agron Crop Sci 194:325333.

Finch-Savage WE, Dent, Clark LJ (2004). Soak condition and temperature following sowing influence the response of maize(Zea mays L.) seeds to on-farm priming (pre-sowing seed soak). Field Crops Res 90:361-374.

Fu JR, Lue XH, Chen RZ, Zhang BZ, Liu ZS, Ki ZS, Cai CY (1988). Osmoconditioning of peanut (Arachis hypogaea L.) seeds with PEG to improve vigour and some biochemical activities. Seed Sci Technol 16:197-212.

Giri GS, Schilinger WF (2003). Seed priming winter wheat for germination, emergence and yield. Crop Sci 43:2135-2141.

Harris D, Joshi A, Khan PA, Gothkar P, Sodhi PS (1999). Onfarm seed priming in semi arid agriculture: Development and evaluation in maize, rice and chickpea in India using participatory methods. Exper Agric 35:15-29.

Hartz TK, Caprile J (1995). Germination of sh2 sweet corn following seed disinfestations, solid matrix priming and microbial seed treatment. Horticulture Sci 30:1400-1402.

Heydecker W, Coolbear P (1978). Seed treatment for improved performance: Survey and attempted prognosis. Seed Sci Technol 5:353-425.

Hur SN (1991). Effect of osmoconditioning on the productivity of Italian ryegrass and sorghum under suboptimal conditions. Korean J Animal Sci 33:101-105.

ISTA, (1996). Rules for Seed Testing. International Seed Testing Association. Seed Sci. Technol. Zurich, Switzerland.

Janmohammadi M, Moradi Dezfuli P, Sharifzadeh F (2009) Seed invigouration techniques to improve germination and early growth of inbred lines of maize under salinity and drought stress. Genetic Appl Plant Physiol 34(3-4):215-226.

Jie L, Gong She L, Dong Mei O, Fang Fang L, En Hua W (2002). Effect of PEG on germination and active oxygen metabolism in wildrye (Leymus chinensis) seeds. Acta Prataculturae Sin 11:59-64.

Khalil S, Moursy HA (1983). Changes in some germination, morphological, physiological and reproductive characters of tomato plant as influenced by heat treatment of seeds. Ain Shams University. Annual Agric Sci 28:1099-1121.

Khalil SK, Mexal JG, Ortiz M (1997). Osmotic priming hastens 
germination and improves seedling size of Pinus brutia var. eldarica. Tree Planters Not 48:24-27.

Khan AA (1992). Preplant physiological seed conditioning. Horticultural Rev 13:131-181.

Kaur S, Gupta AK, Kaur N (2005). Seed priming increases crop yield possibly by modulating enzymes of sucrose metabolism in chickpea. J Agron Crop Sci 191:81-87.

Kaya MD, Okcu G, Atak M, Cikili Y, Kolsarici O (2006). Seed treatments to overcome salt and drought stress during germination in sunflower (Helianthus annuus L.). European J Agron 24:291-295.

Kumar A, Gangwar JS, Prasad SC, Harris D (2002). On-farm seed priming increases yield of direct sown finger millet in India. The Internat Sorghum Millets Newsland 43:90-92.

Liu Q, Hilhorst HWM, Groot SPC, Bino RJ (1997). Amounts of nuclearDNA and internal morphology of gibberellin and abscisic acid deficient tomato (Lycopersicon esculentum Mill.) seeds during maturation, imbibition and germination. Annals Bot 79:161-168.

Michel BE, Kaufmann MR (1973). The osmotic potential of polyethylene glycol 6000. Plant Physiol 51:914-916.

Moradi Dezfuli P, Sharif-zadeh F, Janmohammadi M (2008) Influence of priming techniques on seed germination behavior of maize inbred lines (Zea mays L.). ARPN J Agricultural Biol Sci 3(3):22-25

Parera CA, Cantliffe DJ (1994) Pre-sowing seed priming. Horticultural Rev 16:109-141.

Passam HC, Kakouriotis D (1994). The effects of osmoconditioning on the germination, emergence and early plant growth of cucumber under saline conditions. Scienta Hort 57:233-240.
Rouhi HR, Abbasi Surki A, Sharif-Zadeh F, Tavakkol Afshari R, Aboutalebian MA, Ahmadvand G (2011). Study of different priming treatments on germination traits of soybean seed lots. Not Sci Biol 3(1):101-108.

Shivankar RS, Deore DB, Zode NG (2003). Effect of pre-sowing seed treatment on establishment and seed yield of sunflower. J Oilseeds Res 20:299-300.

Singh G, Gill SS, Sandhu KK (1999). Improved performance of muskmelon (Cucumis melo) seeds with osmoconditioning. Acta Agrobotany 52:121-126.

Snap S, Price R, Morton M (2008). Seed priming of winter annualcover crops improves germination and emergence. Agronomy J 100:1-5.

Srinivasan K, Saxena S, Singh BB (1999). Osmo and hydropriming of mustard seeds to improve vigour and some biochemical activities. Seed Sci Technol 27:785-789.

Styer RC, Cantliffe DJ (1983). Evidence of repair processes in onion seed during storage at high seed moisture contents. J Experimental Bot 34:277-282.

Suzuki H, Khan AA (2001). EVective temperatures and duration for seed humidification in snapbean (Phaseolus vulgaris L.). Seed Sci Technol 28:381-389.

Thornton JM, Powell AA (1992). Short term aerated hydration for the improvement of seed quality in Brassica oleracea L. Seed Sci Res 2: 41-49.

Zheng GH, Wilen RW, Slin Kard AE, Gusta LV (1994). Enhacement of Canola seed germination and seedling emergence at two temperature by priming. Crop Sci 34(6):1589-1593. 